\title{
Role of concurrent chemoradiation on locally advanced unresectable adenoid cystic carcinoma
}

\author{
Hyerim $\mathrm{Ha}^{1,2,}$, Bhumsuk Keam ${ }^{1,3}$, Chan-Young Ock ${ }^{1}$, Tae Min Kim, ${ }^{1,3}$ Jin Ho Kim ${ }^{4}$, Eun-Jae Chung ${ }^{5}$, \\ Seong Keun Kwon ${ }^{5}$, Soon-Hyun Ahn' ${ }^{5}$, Hong-Gyun Wu ${ }^{4}$, Myung-Whun Sung 5 , and Dae Seog Heo ${ }^{1,3}$
}

\begin{abstract}
${ }^{1}$ Department of Internal Medicine, Seoul National University Hospital, Seoul; ${ }^{2}$ Department of Internal Medicine, Incheon St. Mary's Hospital, College of Medicine, The Catholic University of Korea, Incheon; ${ }^{3}$ Cancer Research Institute, Seoul National University College of Medicine, Seoul; Departments of ${ }^{4}$ Radiation Oncology and ${ }^{5}$ Otorhinolaryngology, Seoul National University Hospital, Seoul, Korea
\end{abstract}

Received: March 25, 2019

Revised : July 2, 2019

Accepted: July 26, 2019

\section{Correspondence to}

Bhumsuk Keam, M.D.

Department of Internal Medicine, Seoul National University Hospital, 101 Daehak-ro, Jongno-gu, Seoul 0308o, Korea

Tel: +82-2-2072-7215

Fax: +82-2-2072-7379

E-mail: bhumsuk@snu.ac.kr

https://orcid.org/0000-0001-

8196-4247

*Current affiliation:

Department of Internal Medi-

cine, Inha University Hospital,

Incheon, Korea
Background/Aims: Adenoid cystic carcinoma (ACC) is a rare salivary gland tumor characterized by indolence, with a high rate of local recurrence and distant metastasis. This study aimed to investigate the effect of concurrent chemoradiation (CCRT) on locally advanced unresectable ACC.

Methods: We retrospectively analyzed clinical data from 10 patients with pathologically confirmed ACC of the head and neck who received CCRT with cisplatin in Seoul National University Hospital between 2013 and 2018.

Results: Ten patients with unresectable disease at the time of diagnosis or with positive margins after surgical resection received CCRT with weekly cisplatin. Eight patients (80\%) achieved complete remission, of which three later developed distant metastases without local relapse; one patient developed distant metastasis and local relapse. Two patient achieved partial remission without progression. Patients experienced several toxicities, including dry mouth, radiation dermatitis, nausea, and salivary gland inflammation of mostly grade 1 to 2 . Only one patient showed grade 3 oral mucositis. Median relapse-free survival was 34.5 months (95\% confidence interval, 22.8 months to not reached).

Conclusions: CCRT with cisplatin is effective for local control of ACC with manageable toxicity and may be an effective treatment option for locally advanced unresectable ACC.

Keywords: Carcinoma, adenoid cystic; Chemoradiotherapy; Cisplatin

\section{INTRODUCTION}

Adenoid cystic carcinoma (ACC) is a rare malignant tumor that mainly occurs in salivary glands but can develop in various tissues of the body including the trachea, sinus, breast, and lung [1-3]. ACC constitutes 10\% of salivary gland tumors and approximately $1 \%$ of head and neck tumors [4]. ACC progresses slowly but has a high rate of perineural invasion, local recurrence, and indolent distant metastasis $[5,6]$.

The treatment of choice for ACC without distant metastasis is complete surgical excision with negative resection margin [7]. However, in locally advanced cases, ACC frequently invades the surrounding anatomy of the head and neck, and wide excision with sufficient margin is often difficult [6]. Patients with ACC respond 
well to radiotherapy, and radical radiation therapy (RT) has been applied in unresectable cases [8]. It is not clear whether radical RT alone improves survival outcomes or relieve symptoms in locally advanced ACC. Several cases have been reported with promising results from concurrent chemoradiation (CCRT) [9,10].

Cisplatin is well known for its role as a radiosensitizer for several malignancies, owing to its interference with DNA double-strand break repair [11,12]. Theoretically, radical CCRT may be more effective than radical RT alone, for local disease control in ACC. However, the effect of CCRT on ACC has not been fully established.

This study aimed to evaluate the efficacy and feasibility of CCRT in locally advanced unresectable ACC.

\section{METHODS}

\section{Patient population}

Medical records were reviewed retrospectively for patients who were diagnosed with locally advanced unresectable ACC and treated with CCRT at Seoul National University Hospital between 2013 and 2018. Diagnosis was confirmed pathologically. TNM stage of patients was established according to the 8th edition of the American Joint Committee on Cancer (AJCC). In brief, the AJCC 8th edition defines stage $\mathrm{T}_{3}$ as a tumor invading soft tissue and stage $\mathrm{T}_{4}$ as a tumor invading a nearby structure, such as the jaw bone, skull, skin, or nerves. Each case was presented for multidisciplinary evaluation before making recommendations for definitive therapy. Unresectable status was determined by the multidisciplinary team, comprising head and neck cancer experts from Seoul National University Hospital.

Adult patients aged 18 years or older with more than one measurable lesion according to the Response Evaluation Criteria for Solid Tumors (RECIST 1.1) [13], an Eastern Cooperative Oncology Group performance status (ECOG PS) score of o to 2, and adequate hematologic, hepatic, and renal function were included for analysis.

\section{Treatment and analysis}

CCRT consisted of conventional fractionated radiotherapy for the primary tumor \pm regional lymph nodes, with concurrent intravenous cisplatin chemotherapy. Nine patients received RT using volumetric-modulated arc therapy. Only one patient received intensity-modulated radiotherapy with simultaneous integrated boost. Four patients received 21 to 33 fractionated doses of RT to only the primary tumor site. Six patients received 30 fractionated doses of RT to both the primary site and around the tumor structure. Details about dose and fractions are summarized in Table 1 . Nine patients received $35 \mathrm{mg} / \mathrm{m}^{2}$ of intravenous cisplatin for 6 cycles every week, and one patient received $100 \mathrm{mg} / \mathrm{m}^{2}$ of cisplatin for 3 cycles every 3 weeks. Responses were evaluated according to RECIST 1.1, and toxicity was assessed according to National Cancer Institute Common Terminology Criteria for Adverse Events (NCI CTCAE v5.0).

Relapse-free survival (RFS) was defined as the time from the first day of CCRT to the date of relapse, disease progression, distant metastasis, or death. Median RFS, overall survival (OS), and median follow-up period were calculated using the Kaplan-Meier method.

\section{Ethics}

The study protocol was reviewed and approved by the Institutional Review Board of Seoul National University Hospital (No.: H-1812-059-993). All studies were conducted according to guidelines for biomedical research and Declaration of Helsinki. The requirement for informed consent from patients was waived because this study was retrospective.

\section{RESULTS}

\section{Patient characteristics}

Three patients were male and seven were female. Median age at the time of diagnosis was 55 years (range, 46.8 to 60.6). Seven patients were ineligible for resection because of bulky mass, perineural invasion, and inoperable location. Two patients declined surgery due to surgical morbidity. The remaining patient received surgical resection with positive margins, and the tumor was subsequently determined to be unresectable by the multidisciplinary team because further resection was impossible. Table 1 lists the reasons for unresectable status.

The primary tumor site was the parotid gland in three patients, the palate in two patients, and the sublingual salivary gland, buccal, maxillary sinus, external auditory tract, and trachea in the remaining patients. Only 


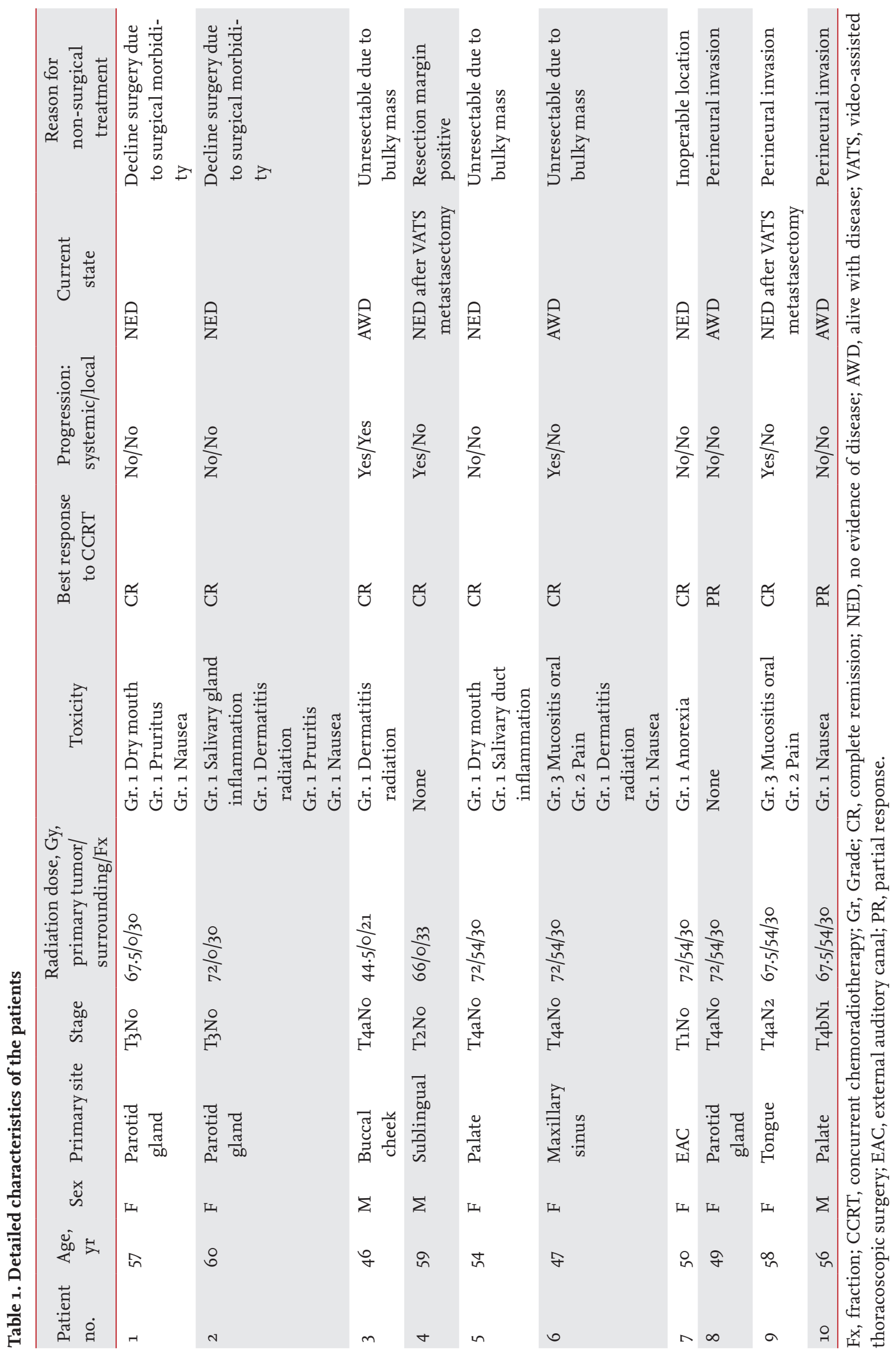




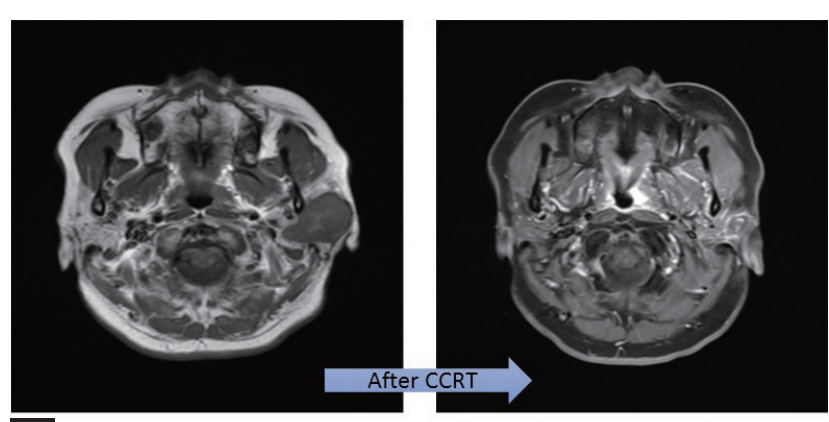

A

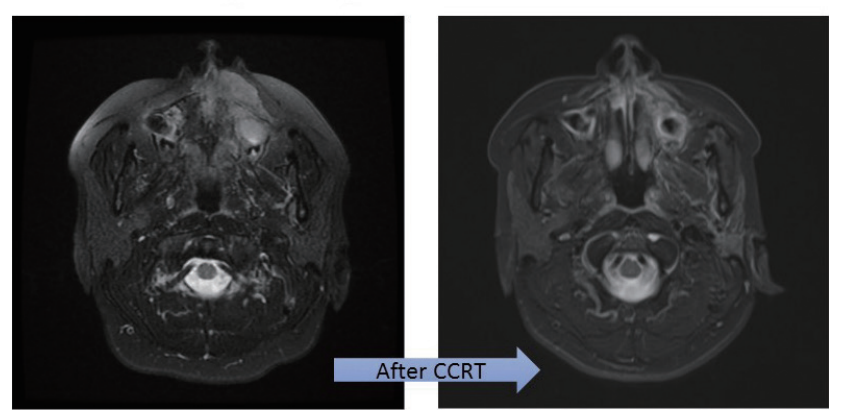

B

Figure 1. Representative image before and after concurrent chemoradiation. (A) Patient No. 1: light parotid gland mass disappeared after concurrent chemoradiotherapy (CCRT). (B) Patient No. 6: adenoid cystic carcinoma of maxillary sinus disappeared after CCRT.

one patient had positive node involvement, and none showed distant metastasis. The median follow-up period was 35.7 months (range, 8.3 to 68.8 ).

\section{Treatment outcomes}

Patients received a median of 69.8 Gy (range, 44.5 to 72.0) of radiation. Four patients received radiation at the primary tumor site, and six patients received radiation at both the primary site and the surrounding structure. Eight patients achieved complete remission (CR), and two patients achieved partial remission(PR)(Fig. 1). Local recurrence was observed in one patient who received the lowest dose of radiation. Four patients developed lung metastasis after CCRT (Table 1). One patient received radiofrequency ablation, and two patients underwent video-assisted thoracoscopic surgery (VATS) for single lung metastasis. One patient with multiple lung metastases underwent systemic therapy with axitinib. Among four patients with metastasis, two showed progression of lung lesion without local progression of the primary tumor, and the two patients who underwent VATS

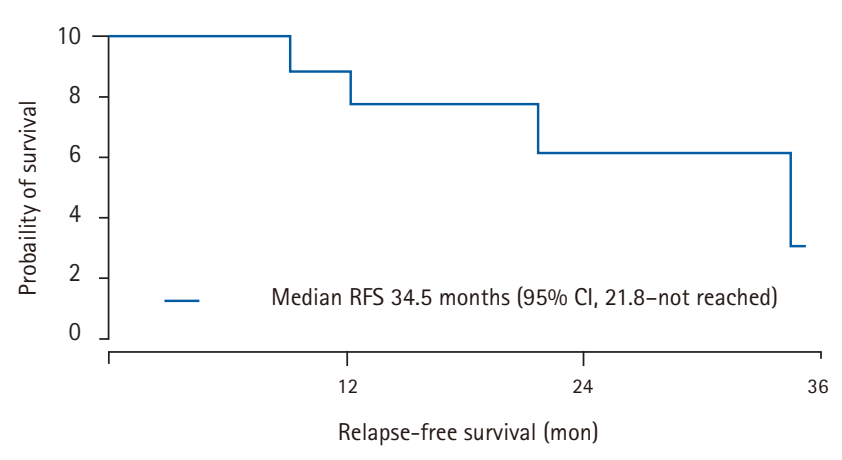

Figure 2. Relapse-free survival after concurrent chemoradiation. RFS, relapse-free survival; CI, confidence interval.

retained no evidence of disease. All patients were alive. Median RFS was 34.5 months (95\% confidence interval, 21.8 to not reached) (Fig. 2). Median OS was not reached (95\% confidence interval, not reached to not reached).

\section{Adverse events}

The most common toxicities of CCRT with cisplatin were nausea $(n=4)$ and radiation dermatitis $(n=3)$. Dry mouth (xerostomia), pruritis, and salivary gland inflammation were reported in two patients, respectively. Two patients showed grade 3 oral mucositis (Table 2) $[10,14,15]$. All toxicities were tolerable.

\section{DISCUSSION}

In this study, locally advanced unresectable ACC patients receiving radical CCRT had a high CR rate of $80 \%$ with low local recurrence.

The primary treatment for ACC is complete surgical resection, but it is often difficult to perform surgery due to the invasion of the surrounding structures such as the nerve, skull base, and brain $[16,17]$. ACC of the trachea, nasopharynx, or maxillary sinus that are unable to undergo surgery have been treated with radiotherapy [18]. The effect of definitive RT for unresectable cases has been reported from several retrospective studies, with a response rate of approximately $40 \%$ and RFS of approximately 30 months [19-21]. However, it is difficult to obtain long-term remission in unresectable cases by RT alone [22]. There have been a small number of case reports regarding the effects ofCCRT, indicating a tolerable local control rate for ACC [10,23-25]. Ten patients di- 
Table 2. Summarization of previous studies about concurrent chemoradiation in ACC

\begin{tabular}{|c|c|c|c|c|}
\hline Study & $\begin{array}{c}\text { No. of } \\
\text { patients }\end{array}$ & Treatment & Response & Survival \\
\hline Haddard et al. (2006) [14] & 5 & $\begin{array}{l}\text { CCRT with carboplatin/ } \\
\text { paclitaxel }\end{array}$ & $4(80 \%) \mathrm{CR}$ & $\begin{array}{l}3 \text { yr OS } 100 \% \\
3 \text { yr LC 100\% }\end{array}$ \\
\hline Gomez et al. (2008) [15] & 5 & $\begin{array}{l}\text { CCRT with cisplatin or } \\
\text { carboplatin/paclitaxel }\end{array}$ & $\begin{array}{l}2(40 \%) \text { CR } \\
2(40 \%) \text { local failure after } 1.5 \mathrm{yr} \\
1(20 \%) \text { systemic failure after } 7 \mathrm{yr}\end{array}$ & No data \\
\hline Sanmant et al. (2012) [10] & 16 & $\begin{array}{l}\text { CCRT with cisplatin or } \\
\text { carboplatin }\end{array}$ & $\begin{array}{l}8(50 \%) \mathrm{CR} \\
7(44 \%) \mathrm{PR} \\
2(13 \%) \mathrm{SD}\end{array}$ & $\begin{array}{l}5 \text { yr OS } 87 \% \\
5 \text { yr LRFS 61\% }\end{array}$ \\
\hline
\end{tabular}

ACC, adenoid cystic carcinoma; CCRT, concurrent chemoradiotherapy; OS, overall survival; LC, local control; CR, complete remission; PR, partial response; SD, stable disease; LRFS, local relapse free survival.

agnosed with ACC received CCRT in our study. Among them, eight patients $(80 \%)$ achieved CR, experiencing long-term locoregional control with no local progression or relapse, and the remaining patients showed PR. Our results are in line with those of previous reports [2325], suggesting that CCRT could be more effective than radical RT alone. CCRT with cisplatin may be a good treatment option for patients with unresectable ACC.

ACC of the salivary gland is associated with an increased risk of a positive margin following resection, compared to adenocarcinoma, and is therefore associated with poor prognosis [26]. Although one study reported that surgery alone provided long-term survival rates comparable to those of surgery plus radiotherapy, others found that radiotherapy was effective in cases involving positive margins [27-29]. In our study, a patient receiving postoperative CCRT achieved CR, followed by systemic relapse but not local recurrence. CCRT can therefore be expected to affect local disease control following surgery.

Cisplatin is used as a radiosensitizer in CCRT for many cancers. However, CCRT with cisplatin often fails to proceed as planned due to toxicity [30,31]. Recently, platinum-based CCRT has been reported to be effective for nasopharyngeal cancer [32,33]. All patients in this study received cisplatin with radiotherapy. Nine patients were injected with cisplatin dose of $35 \mathrm{mg} / \mathrm{m}^{2}$ in 6 cycles per week. One patient received high-dose cisplatin every 3 weeks. Patients experienced toxicities including dermatitis, xerostomia, nausea, and changes in taste, but most toxicities were tolerable at grade 1 or 2. CCRT with cisplatin is therefore effective with tolerable toxicity.
Our study has several limitations. This study was retrospective and analyzed a small number of just 10 cases; all cases were from a single center, and CCRT was not compared with radical radiotherapy. However, considering the rare incidence of ACC, a small number of studies will still be meaningful. No previous studies have analyzed the effect of CCRT with cisplatin in the same clinical setting, so our study will help select CCRT as a treatment option.

In conclusion, this study suggests that CCRT with cisplatin may be an effective treatment option with manageable toxicity for locally advanced unresectable ACC. Large-cohort prospective studies are needed to confirm efficacy, and a randomized trial to compare CCRT with radical $\mathrm{RT}$ alone is warranted.

\section{KEY MESSAGE}

1. Concurrent chemoradiotherapy (CCRT) with cisplatin provides a good local control rate with low recurrence in locally advanced unresectable adenoid cystic carcinoma (ACC).

2. CCRT with cisplatin has manageable toxicity in locally advanced unresectable ACC.

\section{Conflict of interest}

No potential conflict of interest relevant to this article was reported.

\section{Acknowledgments}

We thank the patients included in the current study. This 
study was supported by a grant from the Korea Health Technology R\&D Project "Strategic Center of Cell and Bio Therapy for Heart, Diabetes \& Cancer" through the Korea Health Industry Development Institute (KHIDI), funded by the Ministry of Health and Welfare (MHW), Republic of Korea (grant number $\left.\mathrm{HI}_{17} \mathrm{C} 2085\right)$.

\section{REFERENCES}

1. Spiro RH. Salivary neoplasms: overview of a 35-year experience with 2,807 patients. Head Neck Surg 1986;8:177-184.

2. Leeming R, Jenkins M, Mendelsohn G. Adenoid cystic carcinoma of the breast. Arch Surg 1992;127:233-235.

3. Bennett AK, Mills SE, Wick MR. Adenoid cystic carcinoma of the lung. Pathol Case Rev 2004;9:253-258.

4. Spiro RH, Huvos AG, Strong EW. Adenoid cystic carcinoma of salivary origin. A clinicopathologic study of 242 cases. Am J Surg 1974;128:512-520.

5. Ju J, Li Y, Chai J, et al. The role of perineural invasion on head and neck adenoid cystic carcinoma prognosis: a systematic review and meta-analysis. Oral Surg Oral Med Oral Pathol Oral Radiol 2016;122:691-701.

6. Singh FM, Mak SY, Bonington SC. Patterns of spread of head and neck adenoid cystic carcinoma. Clin Radiol 2015;70:644-653.

7. Spiro RH, Huvos AG. Stage means more than grade in adenoid cystic carcinoma. Am J Surg 1992;164:623-628.

8. Mendenhall WM, Morris CG, Amdur RJ, Werning JW, Hinerman RW, Villaret DB. Radiotherapy alone or combined with surgery for adenoid cystic carcinoma of the head and neck. Head Neck 2004;26:154-162.

9. Maruya S, Namba A, Matsubara A, et al. Salivary gland carcinoma treated with concomitant chemoradiation with intraarterial cisplatin and docetaxel. Int J Clin Oncol 2006;11:403-406.

10. Samant S, van den Brekel MW, Kies MS, et al. Concurrent chemoradiation for adenoid cystic carcinoma of the head and neck. Head Neck 2012;34:1263-1268.

11. Groen HJ, Sleijfer S, Meijer C, et al. Carboplatin- and cisplatin-induced potentiation of moderate-dose radiation cytotoxicity in human lung cancer cell lines. Br J Cancer 1995;72:1406-1411.

12. Sears CR, Cooney SA, Chin-Sinex H, Mendonca MS, Turchi JJ. DNA damage response (DDR) pathway engagement in cisplatin radiosensitization of non-small cell lung can- cer. DNA Repair (Amst) 2016;40:35-46.

13. Eisenhauer EA, Therasse P, Bogaerts J, et al. New response evaluation criteria in solid tumours: revised RECIST guideline (version 1.1). Eur J Cancer 2009;45:228-247.

14. Haddad RI, Posner MR, Busse PM, et al. Chemoradiotherapy for adenoid cystic carcinoma: preliminary results of an organ sparing approach. Am J Clin Oncol 2006;29:153-157.

15. Gomez DR, Hoppe BS, Wolden SL, et al. Outcomes and prognostic variables in adenoid cystic carcinoma of the head and neck: a recent experience. Int J Radiat Oncol Biol Phys 2008;70:1365-1372.

16. Kokemueller H, Eckardt A, Brachvogel P, Hausamen JE. Adenoid cystic carcinoma of the head and neck: a 20 years experience. Int J Oral Maxillofac Surg 2004;33:25-31.

17. Ellington CL, Goodman M, Kono SA, et al. Adenoid cystic carcinoma of the head and neck: incidence and survival trends based on 1973-2007 Surveillance, Epidemiology, and End Results data. Cancer 2012;118:4444-4451.

18. Bhandari V, Ladia DD, Kausar M, Varma A. Definitive radiotherapy for inoperable adenoid cystic carcinoma of the trachea: a rare case report. Lung India 2017;34:73-75.

19. Bhattasali O, Holliday E, Kies MS, et al. Definitive proton radiation therapy and concurrent cisplatin for unresectable head and neck adenoid cystic carcinoma: a series of 9 cases and a critical review of the literature. Head Neck 2016;38 Suppl 1:E1472-E148o.

20. Douglas JG, Laramore GE, Austin-Seymour M, Koh W, Stelzer K, Griffin TW. Treatment of locally advanced adenoid cystic carcinoma of the head and neck with neutron radiotherapy. Int J Radiat Oncol Biol Phys 2000;46:551-557.

21. Cerda T, Sun XS, Vignot S, et al. A rationale for chemoradiation (vs radiotherapy) in salivary gland cancers? On behalf of the REFCOR (French rare head and neck cancer network). Crit Rev Oncol Hematol 2014;91:142-158.

22. Chopra S, Kamdar DP, Cohen DS, et al. Outcomes of nonsurgical management of locally advanced carcinomas of the sinonasal cavity. Laryngoscope 2017;127:855-861.

23. Allen AM, Rabin MS, Reilly JJ, Mentzer SJ. Unresectable adenoid cystic carcinoma of the trachea treated with chemoradiation. J Clin Oncol 2007;25:5521-5523.

24. Rosenberg L, Weissler M, Hayes DN, et al. Concurrent chemoradiotherapy for locoregionally advanced salivary gland malignancies. Head Neck 2012;34:872-876.

25. Kim KS, Wu HG, Sung MW. Radiotherapy as a first-line modality for tongue base adenoid cystic carcinoma: re- 
port of 3 cases. Korean J Head Neck Oncol 2014;30:104108.

26. Morse E, Fujiwara RJT, Judson B, Prasad ML, Mehra S. Positive surgical margins in parotid malignancies: Institutional variation and survival association. Laryngoscope 2019;129:129-137.

27. Li Q, Xu T, Gao JM, et al. Surgery alone provides longterm survival rates comparable to those of surgery plus postoperative radiotherapy for patients with adenoid cystic carcinoma of the palate. Oral Oncol 2011;47:170-173.

28. Dautruche A, Bolle S, Feuvret L, et al. Three-year results after radiotherapy for locally advanced sinonasal adenoid cystic carcinoma, using highly conformational radiotherapy techniques proton therapy and/or tomotherapy. Cancer Radiother 2018;22:411-416.

29. Ko YH, Lee MA, Hong YS, et al. Prognostic factors affecting the clinical outcome of adenoid cystic carcinoma of the head and neck. Jpn J Clin Oncol 2007;37:805-811.

30. Lee AW, Tung SY, Chan AT, et al. Preliminary results of a randomized study (NPC-9902 Trial) on therapeutic gain by concurrent chemotherapy and/or accelerated fractionation for locally advanced nasopharyngeal carcinoma. Int J Radiat Oncol Biol Phys 2006;66:142-151.

31. Lee AW, Lau WH, Tung SY, et al. Preliminary results of a randomized study on therapeutic gain by concurrent chemotherapy for regionally-advanced nasopharyngeal carcinoma: NPC-9901 Trial by the Hong Kong Nasopharyngeal Cancer Study Group. J Clin Oncol 2005;23:69666975 .

32. Yu Y, Liang H, Lv X, et al. Platinum-based concurrent chemotherapy remains the optimal regimen for nasopharyngeal carcinoma: a large institutional-based cohort study from an endemic area. J Cancer Res Clin Oncol 2018;144:2231-2243.

33. Hashmi H, Maqbool A, Ahmed S, Ahmed A, Sheikh K, Ahmed A. Concurrent cisplatin-based chemoradiation in squamous cell carcinoma of cervix. J Coll Physicians Surg Pak 2016;26:302-305. 